\title{
Alcohol as an unconditioned stimulus in human classical conditioning
}

\author{
Steven Glautier ${ }^{1}$, Colin Drummond ${ }^{1}$, Bob Remington ${ }^{2}$ \\ ${ }^{1}$ Addiction Research Unit, National Addiction Centre, Institute of Psychiatry, 4, Windsor Walk, London, SE5 8AF, UK \\ ${ }^{2}$ Department of Psychology, University of Southampton, Highfield, Southampton, S09 5NH, UK
}

Received: 18 October 1993 / Final version: 25 January 1994

\begin{abstract}
Recent experiments with human subjects have shown that drug cues (e.g. sight of beer or needle and syringe) elicit different responses than do neutral stimuli. However, because conditioning has not been carried out in the majority of cases, it is not clear why drug cues have different response eliciting capacities; associative and nonassociative mechanisms may both play a part. In this experiment a counterbalanced differential conditioning procedure was used to isolate the role of associative processes in the development of physiological, behavioural, and subjective conditioned responses to cues for alcohol over the course of repeated conditioning sessions. Twelve healthy volunteers took part in the experiment which involved each subject attending for ten sessions. On physiological measures evidence was found for conditioning of skin conductance and cardiac inter-beat interval responses to cues for alcohol delivery. Over the course of conditioning on behavioural measures of drink consumption there were changes in the rate of consumption and number of sips taken as a function of whether or not the drinks contained alcohol. Finally, on subjective measures, there was a differential change in subjective state in response to alcohol and soft drink expectancy as conditioning progressed.
\end{abstract}

Keywords: Alcohol cues - Drug cues - Physiological responses - Conditioning - Drug like - Drug withdrawal like - Drug opposite

Classical conditioning is the fundamental learning process by which organisms adapt to the relationships between events in their environments. In a typical conditioning experiment an innocuous stimulus is recurrently paired with a more potent unconditioned stimulus (US) and, as a consequence of this pairing, the innocuous stimulus acquires new response eliciting capacities and is termed

The work was supported by programme grant No. 7400 from the Medical Research Council

Correspondence to: S. Glautier a conditioned stimulus (CS). For example, in an experiment using dogs, a ticking metronome can be repeatedly paired with food delivery and, after such pairing, the sound of the metronome elicits new responses from the dog, including salivation (Pavlov 1927). These new responses are collectively termed conditioned responses (CRs). Because of similarities between the sequence of events in conditioning experiments and in drug taking situations, there is a great deal of current interest in the role of classical conditioning in drug taking behaviour (see Niaura et al. 1988; Drummond et al. 1990 and Powell et al. 1990 for recent reviews). To elaborate using alcohol consumption as an example; over the course of repeated drinking episodes various relatively innocuous stimuli (e.g. the sight and smell of beer) are paired with alcohol ingestion which is a potent US. As a result of this contingency these relatively innocuous stimuli are likely to acquire CS properties.

The main theoretical models of the role of CSs in the regulation of drug taking all suggest that the presence of drug CSs will elicit CRs which increase the likelihood of drug use (Wikler and Frank 1948; Siegel 1989 and Stewart et al. 1984). The models differ, however, in terms of the mechanism by which the CR exerts its effects. These conditioning models provide a framework within which it is possible to try to understand and investigate the role of drug CSs in the regulation of drug intake and, in the case of problematic drug use, provide a means of approaching the serious problem of relapse after a period of abstinence. In particular, since repeated presentation of a CS without the US results in extinction of CRs, a theoretical basis for investigating cue exposure treatment procedures can be developed. As yet, cue exposure treatment approaches have met with mixed results. Dawe et al. (1993) and McLellan et al. (1986) found no benefit of cue exposure in opiate addicts, nor did Raw and Russell (1980) when they treated smokers. However, Rankin et al. (1983) and Drummond and Glautier (1994) found that alcoholic patients showed some benefits from the cue exposure approach.

As another means of investigating the role of drug CSs in drug taking, laboratory experiments have been geared to studying subjective, physiological and behavioural 
responses to different kinds of drug cue (see reviews, op. cit.). Presentation of stimuli which have been associated with drug taking has been found to result in different responses when the responses are compared with those elicited by stimuli which do not share the same history of association with drug use. However, the majority of studies have been opportunistic, relying on subjects' extraexperimental conditioning histories, rather than actually conditioning subjects. The reasons for this include the fact that opportunistic studies, especially involving patient populations, are much easier to carry out. Subjects may only have to attend one or two sessions and ethical difficulties associated with giving drugs to patients can be avoided.

However, even though it is much easier to carry out opportunistic studies, they have the serious flaw that it is impossible to be sure why different cues elicit different responses. For instance, in one recent study, alcoholic patients had higher skin conductance (SC) levels and reported more feelings of tension and desire to drink in the presence of alcoholic than soft drinks (Glautier and Drummond 1994). While the alcoholic and soft drink cue undoubtedly had different conditioning histories they may well have had other differences and these may have accounted for the different responses. As an example, it is possible that soft and alcoholic drink cues have different unconditioned properties and others have already found that it is desirable to control for this possibility. McCaul et al. (1989) found that differences in "stimulus intensity" (pepper juice versus water) could result in different physiological responses. Robbins and Ehrman (1992) have suggested various ways to improve the design of these opportunistic studies in order to minimise the difficulties with the interpretation of results but, as they point out, it is clear that the only way to be sure that responses elicited by different drink cues are conditioned responses is to actually carry out a carefully designed conditioning experiment. Conditioning experiments using human subjects, in which responses are studied before, during and after conditioning would provide the most cogent demonstrations of drug conditioning and would add considerably to the strength of the present drug conditioning database.

The objective of the present experiment was to study subjective, physiological and behavioural responses to different kinds of CS over the course of successive conditioning trials in which the CSs were paired with an alcohol US. The experiment was designed to model the kinds of conditioning taking place when alcohol is consumed in order to further our understanding of processes likely to play a part in the development and maintenance of problematic drinking. While the primary focus of the experiment was on the development of CRs to different (visual and gustatory) CSs, it was also possible to study the role of alcohol expectancy. It was thought important to explore the likelihood that different CSs might be preferentially associated with the alcohol US because responses to visual CSs which occur before drug taking begins must be at the heart of any conditioning model of the initiation of drug taking. However, there were three potential reasons for suspecting that visual CSs might not support conditioning as well as gustatory CSs; (a) differences in their salience, (b) differences in their associability with drug effects and (c) differences in their temporal relationships with drug effects.

The distinction between visual and gustatory CSs was also drawn in an attempt to follow up the results of an earlier experiment in our laboratory in which the manner of presentation of the drink cue affected physiological and subjective responses to the cue (Glautier et al. 1992b). In that experiment subjects were asked to either drink or hold drinks which had different histories of association with alcohol. It was found that holding and drinking alcohol associated drinks led to different responses. However, there were several possible explanations for this including unconditioned differences between the drink cues used (it was an opportunistic study) and differences in subject's beliefs about the likelihood of alcohol ingestion (holders would not be expecting alcohol). In the current experiment, subjects were conditioned and their responses to the presentation of drinks were studied over the course of conditioning when subjects fully expected to consume the drinks. After conditioning, responses to the presentation of drinks were examined when subjects had been told they would only be holding the drinks, as opposed to actually drinking them. As well as allowing the study of responses to drink cues plus and minus alcohol expectancy, this procedure provided a conditioning experiment parallel to the many opportunistic studies which have studied responses to subjects holding, rather then actually drinking drinks.

\section{Materials and methods}

\section{Design}

There were two parts to the experiment. In the first part, the "conditioning phase", a differential conditioning procedure was carried out in which different drinks were trained as $\mathrm{CS}+$ and CS - . In the second part, the "hold test phase", subjects merely held $\mathrm{CS}+$ and CS - drinks. Physiological, behavioural and subjective responses to $\mathrm{CS}+$ and $\mathrm{CS}$ - were of interest during both parts. During conditioning and holding all factors were within subject and the following counterbalancing and randomisation was carried out.

Half of the subjects were randomly assigned to be conditioned with a red angostura drink as cue for alcohol $(\mathrm{CS}+=\mathrm{ANG})$ whereas the other half were trained with an alcoholic blue peppermint drink as cue for alcohol (CS $+=P E P)$. Subjects trained with the red angostura mixture as $C S+$ were trained with a blue peppermint drink as soft drink cue (CS - ) whereas those trained with the blue peppermint mixture as $\mathrm{CS}+$ were trained with a red angostura drink as CS - . There were 2 hold test days and subjects were presented with $\mathrm{CS}+$ on the first or second day. Thus, there were four possible conditioning cue/hold test order combinations $(2 \times 2)$ and three replicates of this basic design were conducted, using 12 subjects in all. The eight conditioning sessions (four CS + and four CS - ) were carried out on separate days and a conditioning block was defined as the presentation, on successive experimental sessions, of a CS - and CS + drink. This left four conditioning blocks, each of which had two possible orders; CS + drink on day 1 of the block and CS - drink on day 2 (A) or vice versa (B). Four block/order sequences were used $(\mathrm{ABAB}, \mathrm{BABA}, \mathrm{ABBA}$ or $\mathrm{BAAB})$ and each cell of the basic $2 \times 2$ design (described above) was combined with one of these sequences for all three replicates. The design is illustrated in Table 1 . 
Table 1. Design of the experiment. Three replicates of this design were conducted. ANG = angostura trained as $\mathrm{CS}+$; $\mathrm{PEP}$, peppermint trained as $\mathrm{CS}+$; A, alcohol presented on first day of conditioning block; $\mathrm{B}$, alcohol presented on second day of conditioning block

\begin{tabular}{|c|c|c|c|c|}
\hline $\mathrm{CS}+$ drink & $\mathrm{ANG}$ & ANG & PEP & PEP \\
\hline $\begin{array}{l}\text { Hold test order } \\
\text { Conditioning } \\
\text { pattern }\end{array}$ & $\begin{array}{l}\mathrm{CS}+ \\
\mathrm{ABBA}\end{array} \rightarrow \mathrm{CS}-$ & $\begin{array}{l}\mathrm{CS}-\rightarrow \mathrm{CS}+ \\
\mathrm{BAAB}\end{array}$ & $\begin{array}{l}\mathrm{CS}+\rightarrow \mathrm{CS}- \\
\mathrm{ABAB}\end{array}$ & $\underset{\mathrm{BABA}}{\mathrm{CS}-} \rightarrow \mathrm{CS}+$ \\
\hline
\end{tabular}

Table 2. Subject characteristics. EPQ scores on psychoticism $(P)$, extraversion $(E)$, neuroticism $(N)$ and lie (L) scales. Alcohol consumption in weekly units (one unit $=8 \mathrm{~g}$ ethanol)

\begin{tabular}{lccllll}
\hline$N=12$ & Age & $\begin{array}{l}\text { Weekly } \\
\text { units }\end{array}$ & EPQ P & EPQ E & EPQ N & EPQ L \\
\hline Mean & 30.3 & 24.6 & 5.7 & 12.7 & 10.8 & 5.5 \\
Range & $20-46$ & $8-48$ & $1-12$ & $5-19$ & $3-17$ & $1-12$ \\
Std & 7.7 & 14.5 & 2.9 & 5.9 & 4.3 & 3.8 \\
\hline
\end{tabular}

\section{Subjects}

Twelve male subjects were recruited from the staff and students of the Maudsley Hospital, Institute of Psychiatry and local population by posted notices and personal solicitation. Summary data for the subjects' age, weekly alcohol consumption and scores on the Eysenck Personality Questionnaire (EPQ; Eysenck and Eysenck 1975) are given in Table 2. None reported a history of alcoholrelated problems, was suffering from serious physical illness, or was taking medication at the time of the experiment. Subjects were paid $£ 100$ each for their participation. All subjects gave written informed consent to participate in the study, which was approved by the hospital Ethics Committee.

\section{Setting}

The experiment was conducted in a sound-insulated psychophysiological recording room. Subjects sat in a comfortable chair beside a table upon which drinks and questionnaires for the session were laid out. They were observed through a one-way mirror by the experimenter from an adjacent room housing physiological recording apparatus and computer. The subject wore headphones throughout the experiment so that the experimenter was able to give instructions at appropriate times during the sessions.

\section{Drinks}

Two alcoholic drinks $(9.4 \%$ alcohol) were used; one was a red angostura mixture and one was a blue peppermint mixture. Each alcoholic drink had a carefully matched placebo version so that with a total of four drinks (red angostura soft/blue peppermint soft plus the two alcoholic drinks), it was possible to ensure that different flavours and colours served different CS functions (CS + and CS - ) for different subjects. The exact formulation and method of designing these drinks was described in detail by Glautier et al. (1992a). The alcoholic drinks were those described; the angostura CS - was drink A4 and the peppermint CS - was drink B6. The dose of alcohol used was $0.6 \mathrm{~g} / \mathrm{kg}$ and CS - drinks were of the same volume as the alcoholic drink. All drinks were administered as two separate portions presented in plastic glasses which were hidden under opaque containers on a table beside subjects until appropriate times during the sessions. Whenever a CS - drink was presented, a piece of tissue soaked in $2 \mathrm{ml}$ vodka was tapped under the opaque container in order to provide a smell of alcohol. The purpose was to ensure conditioning would occur to visual and/or taste rather than olfactory cues.

\section{Questionnaires}

Subjects completed a short drinking habits questionnaire, which asked about quantity and frequency of alcohol consumption, and the EPQ on the first of their experimental sessions. During sessions, before and after consuming drinks, subjects completed a mood questionnaire to report their subjective state in the three dimensions of pleasure, arousal and dominance (Mehrabian and Russell 1974). After consumption of drinks subjects rated their intoxication on a $150 \mathrm{~mm}$ visual analogue scale with poles labelled "Not at all intoxicated" and "Very intoxicated".

\section{Physiology and drinking behaviour}

Skin conductance, cardiac inter-beat interval (IBI) and finger pulse volume (FPV) were recorded. The equipment for physiological recording consisted of the following; (a) SC4 skin conductance meter, (b) HR heart rate meter, (c) PT1 finger plethysmograph, (d) MCI/I computer interface and (e) PSV1 power supply all made by Contact Precision Instruments $\mathrm{Ltd}$. This equipment allowed digital recording of physiological signals and direct storage of the signal onto computer disc. All data acquisition and analysis was done using IBM compatible computers. The statistical analyses were carried out using either version 3 or 4.01 of SPSSPC. Silver/silver chloride electrodes were used in conjunction with double stick electrode collars for SC recording, both were from Med Associates Inc. A computer program, written in Pascal, was used to record each sip subjects took of their drinks during training and testing. Each sip was recorded by a key press and the program timed the point at which the subject was instructed to begin drinking, the point at which each sip was taken, and the time the subject finished the drink. The program then calculated summary statistics based on these data (sip count, average sip volume, latency to begin drinking following instruction, and rate of consumption). Finally, breath alcohol level was measured following consumption of alcoholic drinks using a Lion SD-2 Alcometer. The purpose of measuring breath alcohol was twofold. Firstly, it served as a prop to help convince subjects that this was a focus of interest in the experiments. It was considered important that subjects did not know that their responses to the presentation of drinks was being studied. Secondly, breath alcohol was used to actually index the blood alcohol levels.

\section{Procedure}

On initial contact, usually by telephone, subjects were told that the experiment was an investigation into the development of tolerance to the physiological and subjective effects of alcohol involving ten sessions each lasting about $1 \mathrm{~h}$. Subjects were screened to ensure 
they met the study entry criteria and given the following information about the experiment. They were told they would receive alcohol on half of the sessions and soft drink on other days but that they would not know what they were to get in advance. They were told that the dose of alcohol would be approximately equivalent to two pints of beer and because it would be consumed quickly that it was likely to make them intoxicated. Therefore they were advised that they should not plan to drive a car for the rest of the day or undertake any activity which might be dangerous if they were impaired. In addition, subjects were asked not to eat or drink for $2 \mathrm{~h}$ before the sessions, not to smoke for $1 \mathrm{~h}$ before sessions, told that they would be paid $£ 100$ on completion of the study and the first appointment was made.

\section{Conditioning phase}

On arrival on day 1 , the rationale and description of the experiment was restated. Subjects signed a consent form, were weighed and asked to complete the drinking habits and EPQ intake questionnaires. The laboratory was prepared while subjects filled out these questionnaires. This involved setting out mood and intoxication questionnaires, and mixing and pouring of drinks. Subjects were then escorted to the laboratory to begin the first session. On all subsequent days, preparations were made before subjects arrived so that they could be escorted directly to the laboratory. Once in the laboratory subjects were asked to wash their hands before being seated and breathalysed. They were then connected to recording apparatus and told to expect either a red angostura (or blue peppermint) alcoholic drink or a blue peppermint (or red angostura) soft drink as appropriate for the session. On day 1 details of procedures were read to subjects verbatim so that subjects knew what to expect and to ensure standardisation between subjects. A full copy of these, including those used on hold test days, is available upon request. The experimenter then left the subject room. After 5 min subjects completed the first mood questionnaire, after $12 \mathrm{~min}$ a 6-min physiological recording began. Two minutes into the recording subjects lifted one of the opaque containers revealing the first drink, 2 min after that subjects took a small sip of the drink and 2 min later subjects were told to consume the drink within a 10 -min period. If a subject had not finished the drink after $10 \mathrm{~min}$ they were asked to do so. Five minutes after finishing the drink subjects completed another mood questionnaire following which the same procedures were repeated for the second drink. Five minutes after finishing the second drink subjects completed the third mood questionnaire and intoxication rating scale. If alcohol had been given subjects were disconnected and breathalysed again after $15 \mathrm{~min}$, otherwise they were disconnected and the session was terminated immediately. Before breath was sampled, subjects rinsed their mouths with water to remove any traces of alcohol still in the mouth.

\section{Hold phase}

Initial procedures on days 9 and 10 were the same as on previous days. At the point at which the experimenter normally told the subjects which drink to expect in the session, the subjects were told that a "movement control" procedure was going to be used for some subjects. In actual fact, all subjects were going to go through the procedure and the experimenter went on to explain in detail what it involved. On the first of the test sessions instructions were read verbatim to subjects, the experimenter then left the recording room. At the 12 -min point a $16-$ min physiological recording began. Two minutes into the recording the experimenter announced "You are going to be in the hold condition". The experimenter had previously explained to subjects that they would be in either the "hold" or "drink" condition. Thus, up until the announcement, subjects were uncertain about what condition they would be in. Subjects had been told that if they were in the drink condition that the session would proceed as usual but that if they were in the hold condition that they would not be drinking the first drink. Instead they were told that they would be asked to "act out drinking" for 10 min (picking up, looking at and lifting the glass to their lips repeatedly). Four minutes into the recording the first container was lifted, 6 min into recording subjects took a small sip of the drink and 8 min into the recording the first hold instruction was issued. Four hold instructions were then repeated at $2-\min$ intervals before subjects were instructed to place the container back over the drink. The session then proceded in exactly the same way as on conditioning days with subjects consuming a second drink. The consumption of the second drink was timed and constituted the drinking speed test. The second drink (drinking speed test drink) was always the alcohol associated drink on days 9 and 10. The first drink was CS + on one of the days and $\mathrm{CS}$ - on the other as per the design.

\section{Data analysis and reduction}

Data from the conditioning phase of the experiment were analysed as follows. Physiological data relating to the first drink were reduced by averaging over each of the first (baseline), third (sight cue alone), and 5 th min (sight cue plus taste cue) of the recording on each training day. To test the hypothesis that conditioning occurred change scores were obtained by subtracting baseline values from the $3 \mathrm{rd}$ and 5 th $\mathrm{min}$ and entering them into $2(\mathrm{CS}+$ versus $\mathrm{CS}-) \times 2$ (block 1 versus block 4$) \times 2$ (sight versus sight plus taste) analyses of covariance (ANCOVAs) using first minute values as covariates of the change score. The purpose of using ANCOVA in this way was to remove any variation in change scores arising from variation in baseline level. Conditioning effects were also tested for by evaluating trends across time in response to $\mathrm{CS}+$ and CS - by fitting polynomial contrasts across levels of BLOCK for sight and sight plus taste data separately. Again ANCOVA was used in assessing these contrasts. Next, in order to test the effects of a conditioning history on alcohol expectancy (CS + versus CS - days), analyses of baseline levels on physiological and subjective data during the conditioning phase were undertaken by fitting polynomial contrasts across levels of BLOCK in 2 (CS + versus $\mathrm{CS}-$ ) $\times 4$ (block 1-4) ANOVAs. Finally, behavioural responses to $\mathrm{CS}+$ and $\mathrm{CS}-$ drinks were studied using $2(\mathrm{CS}+$ versus $\mathrm{CS}-) \times 4$ (block $1-4)$ analyses of variance (ANOVAs). Rate of consumption, latency to begin drinking following instruction and sip count were analysed by fitting polynomial contrasts across levels of BLOCK. In these analyses $\mathrm{CS} \times \mathrm{BLOCK}$ interactions indicate the presence of conditioning.

During the hold phase of the experiment physiological data were reduced by averaging over each of the 1 st (baseline), 5th (sight cue alone), 7 th (sight cue plus taste cue), 9th, 11th, 13th and 15th (alternate minute during the hold procedure) min of the recording. Change scores were obtained by subtracting baseline measures before carrying out 2 (CS + versus $\mathrm{CS}-$ ) $\times 6$ (time points) $\mathrm{AN}$ COVAs using baseline values as covariates of the change scores. Subjective effects of holding CS + and CS - drinks were assessed by comparing changes in scores between mood questionnaires one and two (baseline and immediately after holding) using the scores obtained in the baseline period as covariates. The effects of holding $\mathrm{CS}+$ and $\mathrm{CS}-$ drinks on subsequent consumption (rate of consumption, number of sips and latency to first sip) and intoxicating effects (mood questionnaire, intoxication ratings and breath alcohol levels) of the alcoholic drinking speed test drink were also assessed. Subjective effects, as measured by change in mood between questionnaire 1 and 3 (baseline and 5 min after finishing the drink) were compared on CS + and CS - days using baseline subjective state as covariates whereas intoxication ratings and breath alcohol level on different days had rate of consumption as covariates.

\section{Results}

The effects of alcohol association (CS + or CS - ), conditioning block (1-4), time within experimental sessions and 
sensory modality of drink cue (sight or sight plus taste) are denoted by CS, BLOCK, TIME and MODALITY, respectively, and interactions by " $x$ ". Where the response to the sight cues accompanying the drink are referred to a symbol (s) is used, where the response to the sight plus taste cues is referred to a symbol (t) is used. One subject was dropped from the analysis of hold data because he was given the wrong drinks on the final day (blue peppermint CS - instead of red angostura CS - ).

\section{Conditioning phase}

During the conditioning phase of the experiment, evidence was found for conditioning of physiological responses to initial sight and sight plus taste cues for alcohol delivery on SC but for IBI, evidence for conditioning to the sight plus taste cues for alcohol was absent. For FPV, however, there were no clear signs of conditioning to either sight or the sight plus taste cues for alcohol. The SC and IBI data is illustrated in Figs 1a and b, respectively.

On SC the initial responses (block one) to $\mathrm{CS}+$ (filled symbols) and CS - (empty symbols) were different to the final responses (block four). On block one CS - resulted in a larger response than $\mathrm{CS}+$ and vice versa on block four. These graphical impressions have statistical support. The $2(\mathrm{CS}+$ versus $\mathrm{CS}-) \times 2$ (block 1 versus block 4$) \times 2$ (sight versus sight plus taste) ANCOVA gave the following results. There was a CS $\times$ BLOCK interaction $[F(1,10)=6.24, P=0.032]$ indicating conditioning and this interaction was explored further by separately comparing responses to sight and sight plus taste $\mathrm{CS}+$ and $\mathrm{CS}$ - on blocks one and four, again using ANCOVA. On block one the response to $\mathrm{CS}-(\mathrm{s})$ was almost higher than the response to $\mathrm{CS}+(\mathrm{s})$, but responses to $\mathrm{CS}-(\mathrm{t})$ were not larger than responses to $\mathrm{CS}+(\mathrm{t})[F(1,10)=4.30$, $P=0.065 ; \quad F(1,10)=2.60, P=0.138$, respectively $]$. In contrast, on block four, the response to $\mathrm{CS}+-$ s) was higher than the response to $\mathrm{CS}-(\mathrm{s})$, and $\mathrm{CS}+(\mathrm{t})$ was almost larger than CS - (t) $[F(1,10)=18.93, P=0.001$; $F(1,10)=4.38, P=0.064$, respectively.

On IBI, the initial responses (block one) to $\mathrm{CS}+(\mathrm{s})$ and $C S-(s)$ were different to the final responses (block four) but this was not the case for CS $+(\mathrm{t})$ and $\mathrm{CS}-(\mathrm{t})$. On block one $\mathrm{CS}+(\mathrm{s})$ resulted in a smaller IBI response than did $\mathrm{CS}-(\mathrm{s})$, and vice versa on block four. However, for $\mathrm{CS}+(\mathrm{t})$ the response was larger than the response to $\mathrm{CS}-(\mathrm{t})$ on block one and continued to be so on block four. Again these graphical impressions have statistical support. A $2(\mathrm{CS}+$ versus $\mathrm{CS}-) \times 2$ (block 1 versus block 4) $\times 2$ (sight versus sight plus taste) ANCOVA gave the following result. There was a significant CS $\times$ BLOCK $\times$ MODALITY interaction $[F(1,11)=$ $4.95, P=0.048]$ which was given overall precedence in the interpretation of these data. The interaction was explored further by separately comparing responses to sight and sight plus gustatory $\mathrm{CS}+(\mathrm{s})$ and $\mathrm{CS}-$ (s) on blocks one and four, again using ANCOVA. On block one, IBI change in response to $\mathrm{CS}-(\mathrm{s})$ was almost larger than the response to $\mathrm{CS}+$ (s) $[F(1,10)=3.76, P=0.081]$ but the response to $\mathrm{CS}-(t)$ was not significantly different to $\mathrm{CS}+(\mathrm{t}) \quad[F(1,10)=1.12, P=0.315]$. In contrast, on
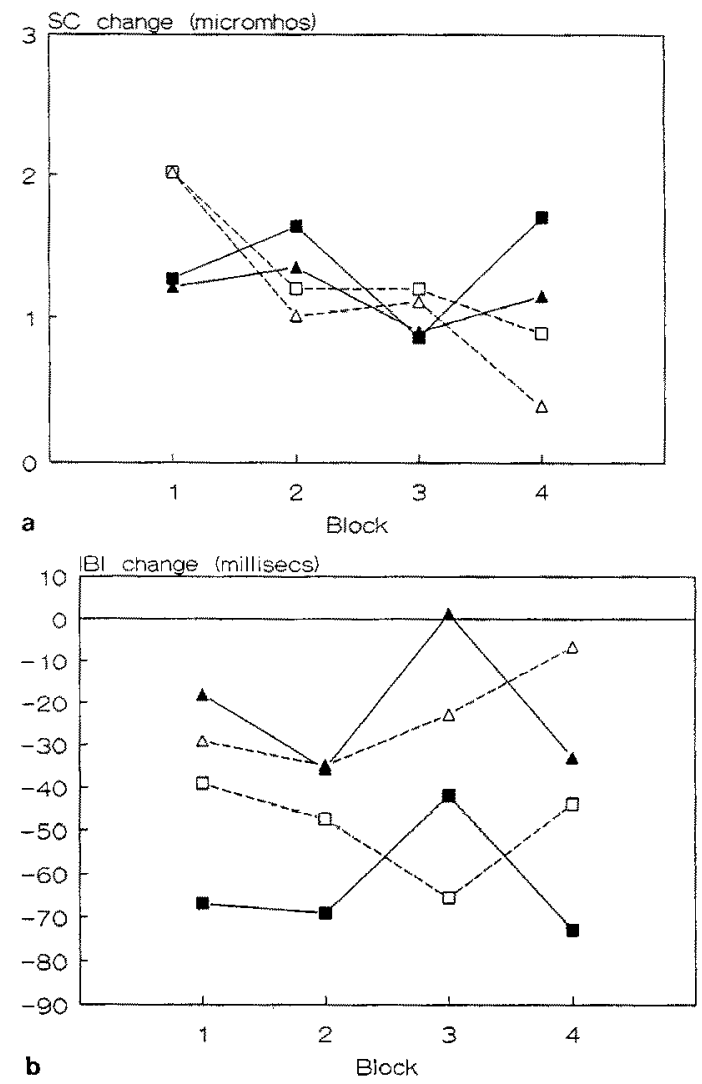

Fig. 1a,b. Conditioning on SC and IBI. SC and IBI changes in response to the sight and sight plus taste of CS + and CS - drinks across conditioning blocks. $-\boldsymbol{\Lambda}-, \mathrm{CS}^{+}$sight; $\cdots-\triangle-\ldots, \mathrm{CS}^{-}$sight; $-\mathbf{- 1}-\mathrm{CS}^{+}$taste; - - $\square--, \mathrm{CS}^{-}$taste

block four, the response to $\mathrm{CS}+(\mathrm{s})$ was almost larger than the response to $\mathrm{CS}-(\mathrm{s})[F(1,10)=3.70, P=0.083]$, whereas again response to CS - (t) was not significantly different to the response to $\mathrm{CS}+(\mathrm{t})[F(1,10)=2.96$, $P=0.116]$.

There were no $\mathrm{CS} \times \mathrm{BLOCK}$ or $\mathrm{CS} \times \mathrm{BLOCK} \times \mathrm{MO}$ DALITY interactions on FPV $[F s<1]$.

Table 3 shows the results of ANCOVAs in which polynomial contrasts were fitted across levels of block for sight and sight plus taste responses on SC and IBI. For $\mathrm{SC}$, there was a significant $\mathrm{CS} \times \mathrm{BLOCK}$ interaction on linear and cubic trends on the sight and sight plus taste responses and this is further evidence of conditioning. However, Roy-Bargmann stepdown $F$-tests, which assess higher order trends (quadratic and cubic) while controlling for lower order trends, resulted in a loss of significance for the $\mathrm{CS}(\mathrm{s}) \times \mathrm{BLOCK}$ and $\mathrm{CS}(\mathrm{t}) \times \mathrm{BLOCK}$ cubic trends $[F(1,6)=3.27, P=0.121 ; F(1,6)=4.49, P=0.078$, respectively]. There was no linear trend across blocks on $\mathrm{CS}+(\mathrm{s})$ considered alone but the linear trend across blocks for CS - (s) was highly significant $[F(1,8)=1.25$, $P=0.296 ; F(1,8)=13.67, P=0.006$, respectively $]$. A similar pattern held when taste responses were considered. There was no linear trend for $\mathrm{CS}+(\mathrm{t})$ responses but a highly significant trend for $\mathrm{CS}-(\mathrm{t})$ responses $[F(1,8)<1 ; \quad F(1,8)=11.54, \quad P=0.009$, respectively $]$. Turning to IBI, for sight plus taste responses, the significant $\mathrm{CS} \times \mathrm{BLOCK}$ quadratic trend remained 
Table 3. Results of ANCOVAs fitting polynomial contrasts across levels of BLOCK on SC and IBI, for sight and taste responses. Effects marked * are detailed further in the text

\begin{tabular}{lllll}
\hline & \multicolumn{2}{l}{$\begin{array}{l}\text { SC responses } \\
F s(1,8), P\end{array}$} & & \multicolumn{2}{l}{$\begin{array}{l}\text { IBI responses } \\
F \text { S }(1,8), P\end{array}$} \\
\cline { 2 - 3 } & Sight & Taste & Sight & Taste \\
\hline BLOCK $\times$ CS & & & & \\
Linear & $7.22,0.028^{*}$ & $4.10,0.078^{*}$ & $2.86,0.129$ & $F<1$ \\
Quadratic & $3.05,0.119$ & $F<1$ & $F<1$ & $6.89,0.030^{*}$ \\
Cubic & $7.66,0.024^{*}$ & $12.25,0.008^{*}$ & $1.08,0.329$ & $7.35,0.027^{*}$ \\
\hline
\end{tabular}

significant in the stepdown test $[F(1,7)=6.03, P=0.044]$ but the cubic trend interaction lost significance $[F(1,6)=2.97, P=0.136]$. There was a significant quadratic trend across blocks in response to $\mathrm{CS}+(\mathrm{t})$, but not on $\mathrm{CS}-(\mathrm{t})[F(1,8)=5.76, P=0.043 ; \quad F(1,8)=1.68$, $P=0.231]$.

Evidence was also found for conditioning on behavioural measures during the conditioning phase of the experiment. $2 \times 4$ ANOVAs showed conditioning effects as evidenced by a $\mathrm{CS} \times$ BLOCK interaction on both sip count and rate of consumption. Overall, rate of consumption increased while sip count fell over sessions and CS drinks were consumed more quickly, with fewer sips. However, differences between CS + and CS - drinks emerged over time, subjects took fewer sips of the CS drink over the course of sessions (which means sips got larger) and consumed the CS - drink faster. These trends are illustrated in Figs 2 and 3. For rate of consumption there was a significant $\mathrm{CS} \times \mathrm{BLOCK}$ interaction for the linear trend $[F(1,11)=4.62, P=0.055]$. For $\mathrm{CS}+$ drinks alone there were no significant linear, quadratic or cubic trends $[F \mathrm{~S}<1]$, whereas for $\mathrm{CS}-$ drinks there was a significant linear trend $[F(1,11)=5.92, P=0.033]$ $[F \mathrm{~s}<1$ for quadratic and cubic trends]. For sip count there was a significant $\mathrm{CS} \times \mathrm{BLOCK}$ interaction for the linear trend $[F(1,11)=10.56, P=0.008]$. For CS + drinks alone there were no significant linear, quadratic or cubic trends $[F \mathrm{~S}<1]$, whereas for CS - drinks there was a significant linear trend $[F(1,11)=6.41, P=0.028]$, with quadratic and cubic trends also evident $[F(1,11)=3.07$, $P=0.108 ; F(1,11)=3.40, P=0.101]$. Comparison of sip count and rate of consumption for $\mathrm{CS}+$ and $\mathrm{CS}-$ drinks on block one resulted in $F \mathrm{~S}<1$ in both cases; however, on block 4 , fewer sips were taken of the CS drink and it was consumed at a higher rate $[F(1,11)=5.43, \quad P=0.040 ; \quad F(1,11)=5.46, \quad P=0.039$, respectively].

Finally, baseline values for physiological and subjective data during conditioning were entered into $2 \times 4$ ANOVAs as a test of the effects of subjects' conditioning experience with the drinks on expectancy of alcohol. A conditioning effect was present on the arousal sub-scale of the mood questionnaire only and consisted of the following pattern. On block one, when subjects expected soft drink they showed lower levels of arousal than subjects expecting alcohol; however, the reverse was true on block four. The threads in arousol baseline are shown in Fig. 4. Overall, there was a significant CS $\times$ BLOCK interaction for the linear trend $[F(1,11)=9.23$, $P=0.011]$. For $\mathrm{CS}+$ the linear trend was not significant $[F<1]$ whereas for $\mathrm{CS}-$ it was $[F(1,11)=9.97$,

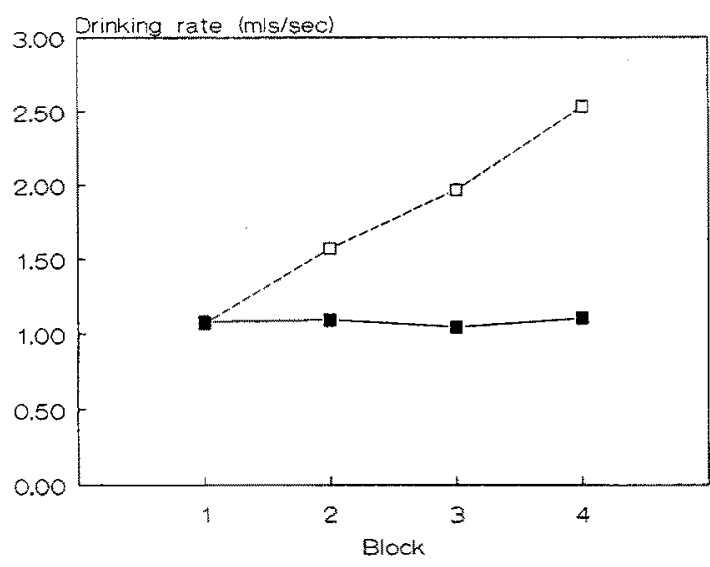

Fig. 2. Rate of consumption of $\mathrm{CS}+$ and $\mathrm{CS}-$ drinks over the course of conditioning blocks. -,$- \mathrm{CS}^{+} ;--\square-\cdots, \mathrm{CS}^{-}$

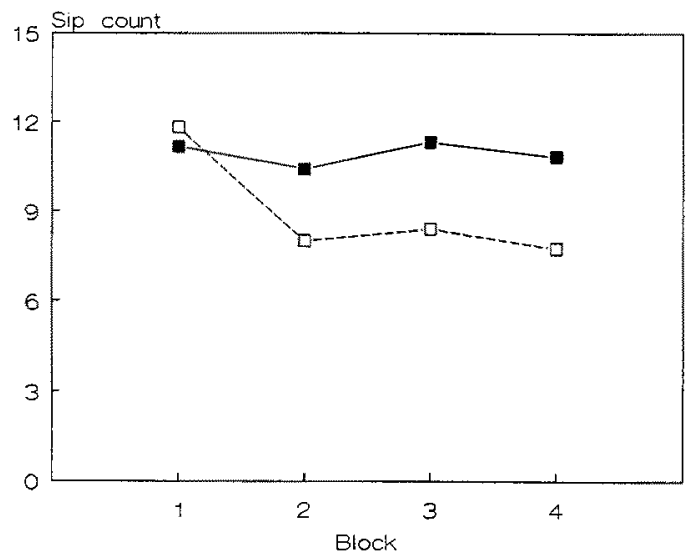

Fig. 3. Number of sips of $\mathrm{CS}+$ and $\mathrm{CS}-$ drinks taken over the course of conditioning blocks. $-\mathbf{E}-\mathrm{CS}^{+} ;--\square--, \mathrm{CS}^{-}$

$P=0.009]$. Comparison of levels at block 1 shows that subjects expecting alcohol have marginally higher levels of arousal $[F(1,11)=2.65, P=0.132]$ whereas the same comparison at block four shows the opposite $[F(1,11)=3.36, P=0.094]$.

\section{Hold test phase}

The $2(\mathrm{CS}+$ versus $\mathrm{CS}-) \times 6$ (time periods) ANCOVAs yielded no CS $\times$ TIME interactions $[F s<1]$ and no effects of CS $[\mathrm{SC}: \quad F(1,9)=2.71, \quad P=0.134 ; \quad F P V$ : $F(1,9)=3.52, P=0.094$; IBI: $F<1]$. In the case of SC and IBI this represents an abolition of differences which 


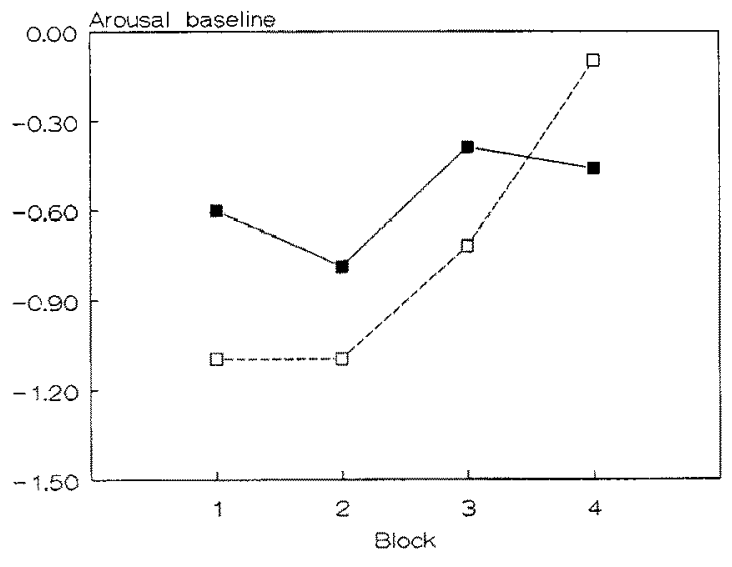

Fig. 4. Baseline levels on arousal scale of the mood questionnaire over the course of conditioning blocks. - - $-\mathrm{CS}^{+} ;--\square--, \mathrm{CS}^{-}$

were present between $\mathrm{CS}+$ and $\mathrm{CS}-$ during the final conditioning block. In the case of FPV, the near significant difference between $\mathrm{CS}+$ and $\mathrm{CS}-$ arose because subjects were more vasoconstricted while holding CS + drinks. There were no significant differences in subjective state following holding $\mathrm{CS}+$ and $\mathrm{CS}-$ drinks $[F(1,9)=1.84, P=0.208$, for the $\mathrm{P}$ scale, $F s<1$ for $A$ and D scales] nor were there any differences in subjective state following consumption of the drinking speed test drink depending upon whether or not subjects had just been holding $\mathrm{CS}+$ and $\mathrm{CS}-$ drinks $[F \mathrm{~S}<1$ for $\mathrm{P}$, $A$ and $D$ scales]. However, subjects who had been holding $\mathrm{CS}+$ drinks had higher breath alcohol levels following consumption of the drinking speed test drink $(46.36 \mathrm{mg} \%$ versus $38.64 \mathrm{mg} \%$ ) and this difference approached significance $[F(1,9)=4.34, P=0.067]$. This was reflected in subject's intoxication ratings; subjects who had been holding CS + drinks rated themselves as more intoxicated following consumption of the drinking speed test drink but this difference was not significant $[F(1,9)=2.71$, $P=0.134]$. Finally, on rate of consumption, number of sips, and latency to first sip there were no significant differences in consumption of the drinking speed test drink when subjects had previously been holding CS + and $\mathrm{CS}-[F \mathrm{~s}<1]$.

\section{Discussion}

The main result of this experiment is the demonstration of classical conditioning on physiological, subjective and behavioural measures using an alcohol US. To summarise, $\mathrm{SC}$ responses to the sight and sight plus taste of $\mathrm{CS}$ - drinks habituated whereas the SC responses to $\mathrm{CS}+$ drinks did not. On IBI, however, the pattern was not quite as clear. There were larger changes in IBI in response to the sight of the CS + drink following conditioning but there was no change in response over the course of conditioning when the sight plus taste CSs were considered. On the subjective measures there was an increasing level of arousal in the baseline periods when subjects expected CS - drinks but when CS + drinks were expected arousal level remained relatively stable.
Finally, on behavioural measures, subjects consumed CS - drinks more quickly and took fewer (therefore larger) sips as conditioning progressed.

The results of the experiment raise a number of issues in relation to (a) theories of the mechanism by which cues affect drug use, (b) conceptualisation of complex nature of cues for alcohol and (c) the procedures which are adopted in experiments of this sort.

It was noted in the introduction that the main theories of cue function all predicted that drug cues would increase drug use motivation although each theory suggested different mechanisms by which this motivational change might come about. Given that responses to $\mathrm{CS}+$ were increases in SC and decreases in IBI then this might constitute evidence in favour of either a model of alcohollike (Stewart et al. 1984) or alcohol-withdrawal-like (Wikler 1948) responses to cues - the withdrawal phase of alcohol's action also results in SC increases and IBI decreases (Niaura et al. 1988). A similar problem with distinguishing between these models is present when the subjective measure of arousal is examined. On this measure, conditioning was manifest as a shift in baseline levels such that when there was an alcohol expectancy, subjects reported lower levels of subjective arousal. This is problematic because alcohol may have bi-phasic effects on subjective, as well as on physiological, measures either as a function of dose or time since ingestion (Tucker et al. 1982). However, if a more general theory of conditioning is used to derive predictions (Wagner 1981), then it is held that the response to a CS will resemble the later phase of the USs action whether or not the particular effect being examined is bi-phasic. Thus, if alcohol's later subjective action really is a depressant one then the predictions for this subjective measure are in keeping with this theory, as are the experimental results reported by Paletta and Wagner (1986). These authors found that responses to a cue for morphine elicited increases in activity which resembled the later phase of morphine's action. So it must be concluded that the results of this experiment do really not help distinguish between the different models of cue function noted in the introduction but may be consistent with a more general conditioning theory in which it is the later phase of a drug's action which is conditioned.

What of the more limited predictions that alcohol cues should increase motivation to drink? Inspection of the behavioural data (Figs 2 and 3) shows that at the outset, $\mathrm{CS}+$ and CS - drinks were more or less equivalent but, over the course of conditioning, the CS - drink was consumed more quickly and fewer (therefore larger) sips of it were taken. Previous workers have taken faster drinking speed as an index of increased motivation to drink (e.g. Rankin et al. 1983) and, unless the opposite is now proposed, this means that the findings of this experiment run against the prediction that alcohol cues should increase motivation to drink. Perhaps this can be understood if the fact that a self administered drug can have aversive properties is recognised (Hunt and Amit 1987). Given the nondependent subject population and the doses used, it is quite possible that subjects learned the relative "safety" of the CS - drinks. As an alternative, the social contingencies of the experiment may have been such that rapid consumption of an alcoholic drink would have been seen as undesirable. 
An additional feature of these behavioural data is the fact that changes occurred to $\mathrm{CS}-$, rather than $\mathrm{CS}+$ drinks. Furthermore, this pattern was seen in the conditioning of the $\mathrm{SC}$ response where a pattern resembling habituation to CS - was seen and in conditioning of the arousal response during the baseline period. Inspection of the sight data in Fig. 2 also shows that IBI responses to the CS - drink showed the largest changes, excepting the unexplained response to $\mathrm{CS}+$ on block three. This all suggests that conditioning in this experiment may have been manifest as a blocking of habituation to the novel drink stimuli. This interpretation is in keeping with accounts of human conditioning which stress the early role of orientation and attentional processes (e.g. Maltzman et al. 1977; Dawson and Schell 1987).

Turning now to the issue of the complex nature of cues for alcohol; because the CS + and CS - drinks used in this experiment differed in terms of their visual appearance as well as their taste, it can be argued that in this respect the drinks were typical of most alcoholic and soft drinks. Therefore, the fact that conditioning occurred to the visual elements of the stimulus complex should serve to dispel any doubts that responses to alcohol cues which occur before drinking begins could play a part in the precipitation of relapse. Indeed it seems likely that visual cues could continue to play a role throughout a drinking episode and, in combination with gustatory cues and cues arising from the conditioned and unconditioned effects of alcohol itself (Hodgson et al. 1979; Stockwell et al. 1982; de Wit and Chutaupe 1993), various kinds of cue may well exert a pervasive influence over the entire sequence of behaviour making up a drinking episode. This point is lent extra weight by the finding that the effects of alcohol expectancy itself changed, apparently as a result of the conditioning procedure. Thus, US expectancy may be treated as a special kind of CS, as well as being conceived of as either a mediator of CR expression (Dawson and Grings 1968; Dawson and Furedy 1976) or as a form of CR itself (Davey 1992; Lovibond et al. 1992). If it is correct to treat expectancy, and perhaps similar cognitive phenomenon, as having the potential of CSs for alcohol use, then the scope for the development and conceptualisation of cue exposure treatment procedures is widened considerably. However, some caution is needed in the interpretation of the data relating to different elements of the cue complex. With regard to the expectancy CS, it should be pointed out that this conditioning procedure was not counterbalanced; that is, no subjects were told to expect alcohol and received soft drink or vice-versa as was the case with the colour/flavour CSs. And, a similar problem may relate to the visual plus gustatory CSs if subjects were able to determine the alcoholic contents of the drinks by some means other than those we had intended. Thus, for example, if the presence of alcohol itself imparted a unique flavour to the drinks then that flavour might have been more salient than other differences between the soft and alcoholic drinks that we arranged. In effect, if subjects conditioned to the taste of alcohol our procedure would not have effectively counterbalanced flavour cues. The failure to condition IBI responses to taste cues argues against this possibility but problems such as this were not ruled out in this experiment.
Finally, the failure to observe differential responding to CS + and CS - on hold test days serves to highlight the potential difficulties for experimental designs of this sort. Because block four differences in response to CS + and CS - were abolished during the hold procedure this suggests that the change in procedure may have resulted in a dishabituation of the CS - response. Of course, this is a post hoc explanation and would be more convincing if it had been shown that responses reinstated their block four levels when normal procedures were reinstated. It is, however, entirely in keeping with the experience of classical conditioning investigators since the earliest times. Pavlov (1927), found the fragility of his animals' conditioned responding to environmental changes a problem for giving experimental demonstrations to visitors as well as a sign of the animals' special sensitivity to changes in their environments. Perhaps the most important thing to be learned from this apparent disruption of conditioning is that alterations in the experimental procedures on critical test days may well result in failure to observe conditioning.

The fact that subjective and physiological measures of activation do not necessarily co-vary is a reflection of the well known problems of an all encompassing activation theory (Lacey 1967).

\section{References}

Davey GCL (1992) An expectancy model of laboratory preparedness effects. J Exp Psychol Gen 121:24-40

Dawe S, Powell J, Richards D, Gossop M, Strang J, Marks I, Gray J (1993) Does post-withdrawal cue exposure improve outcome in opiate addiction? A controlled trial. Addiction 88: 1233-1245

Dawson ME, Furedy JJ (1976) The role of awareness in human differential autonomic classical conditioning: the necessary gate hypothesis. Psychophysiology $13: 50-53$

Dawson ME, Grings WW (1968) Comparison of classical conditioning and relational learning. $J$ Exp Psychol 76:227-231

Dawson ME, Schell AM (1987) Human autonomic and skeletal classical conditioning: the role of conscious cognitive factors. In: Davey $\mathrm{G}$ (ed) Cognitive processes and pavlovian conditioning in humans. Wiley, Chichester

de Wit H, Chutuape MA (1993) Increased ethanol choice in social drinkers following ethanol preload. Behav Pharmacol 4:29-36

Drummond DC, Glautier S (1994) A controlled trial of cue exposure treatment in alcohol dependence. $J$ Consult Clin Psychol (in press)

Drummond DC, Cooper T, Glautier SP (1990) Conditioned learning in alcohol dependence: implications for cue exposure treatment. Br J Addict 85:725-743

Eysenck HJ, Eysenck SBJ (1975) Manual of the Eysenck personality questionaire. Hodder and Stoughton, London

Glautier S, Drummond DC (1994) Alcohol dependence and cue reactivity. J Stud Alcohol 55:224-229

Glautier S, Taylor C, Remington B (1992a) A method for producing alcohol placebos. Br J Addict 87:303-308

Glautier S, Drummond DC, Remington B (1992b) Different drink cues elicit different physiological responses in non-dependent drinkers. Psychopharmacology 106: 550-554

Hodgson R, Rankin H, Stockwell T (1979) Alcohol dependence and the priming effect. Behav Res Ther 17:397-387

Hunt T, Amit Z (1987) Conditioned taste aversion induced by self administered drugs: paradox revisited. Neurosci Biobehav Rey 11: $107-130$ 
Lacey JI (1967) Somatic response patterning and stress: some revisions of activation theory. In: Appley $\mathrm{MH}$, Trumbull $\mathrm{R}$ (eds) Psychological stress. Appleton-Century-Crofts, New York

Lovibond PF, Siddle DAT, Bond N (1992) Insensitivity to stimulus validity in human classical conditioning. Q J Exp Psychol [B] $40: 377-410$

Maltzman I, Gould J, Barnett OJ, Raskin DC, Wolff C (1977) Classical conditioning components of the orienting reflex to words using innocuous and noxious unconditioned stimuli under different CS-US intervals. J Exp Psychol Gen $106: 185-212$

McCaul ME, Turkkan JS, Stitzer ML (1989) Psychophysiological effects of alcohol related stimuli: I. the role of stimulus intensity. Alcohol Clin Exp Res 13:386-391

McLellen AT, Childress AR, Ehrman R, O'Brien CP (1986) Extinguishing conditioned responses during opiate dependence treatment: turning laboratory findings into clinical procedures. J Subst Abuse Treat 3:33-40

Mehrabian A, Russell JA (1974) Approach to environmental psychology. MIT Press, Cambridge

Niaura RS, Rosenhow DJ, Binkoff JA, Monti PM, Pedraza M, Abrams DB (1988) Relevance of cue reactivity to understanding alcohol and smoking relapse. J Abnorm Psychol 97:133-152

Paletta MS, Wagner AR (1986) Development of context specific tolerance to morphine: support for a dual process interpretation. Behay Neurosci 100:611-623

Pavlov IP (1927) Conditioned reflexes. Dover Publications Inc., 1960 , New York

Powell J, Gray JA, Bradley BP, Kasvikis Y, Strang J, Barrat L, Marks I (1990) The effects of exposure to drug-related cues in detoxified opiate addicts: a theoretical review and some new data. Addict Behay 15:339-345

Rankin H, Hodgson R, Stockwell T (1983) Cue exposure and response prevention with alcoholics: a controlled trial. Behav Res Ther 21:435-446

Raw M, Russell MAH (1980) Rapid smoking, cue exposure and support in the modification of smoking. Behav Res Ther $18: 363-372$

Robbins SJ, Ehrman RN (1992) Designing studies of drug conditioning in humans. Psychopharmacology 106:143-153

Siegel S (1989) Pharmacological conditioning and drug effects. In: Goudie AJ, Emmett-Oglesby MW (eds) Psychoactive drugs: tolerance and sensitisation. Humana Press, Clifton, New Jersey, pp 115-180

Stewart J, de Wit H, Eikelboom R (1984) Role of unconditioned and conditioned drug effects in the self administration of opiates and stimulants. Psychol Rev $91: 251-268$

Stockwell TR, Hodgson RJ, Rankin HJ, Taylor C (1982) Alcohol dependence, beliefs and the priming effect. Behav Res Ther $20: 513-522$

Tucker JA, Vuchinich RA, Sobell MB (1982) Alcohol's effects on human emotions: a review of the stimulation/depression hypothesis. Int J Addict 17:155-180

Wagner AR (1981) SOP: a model of automatic memory processing in animal behavior. In: Spear NE, Miller RR (eds) Information processing in animals: memory mechanisms. Laurence Erlbaum, Hillsdale, New Jersey, pp 5-47

Wikler A, Frank K (1948) Hindlimb reflexes of chronic spinal dogs during cycles of addiction to morphine and methadone. J Pharmacol Exp Ther $94: 382-400$ 